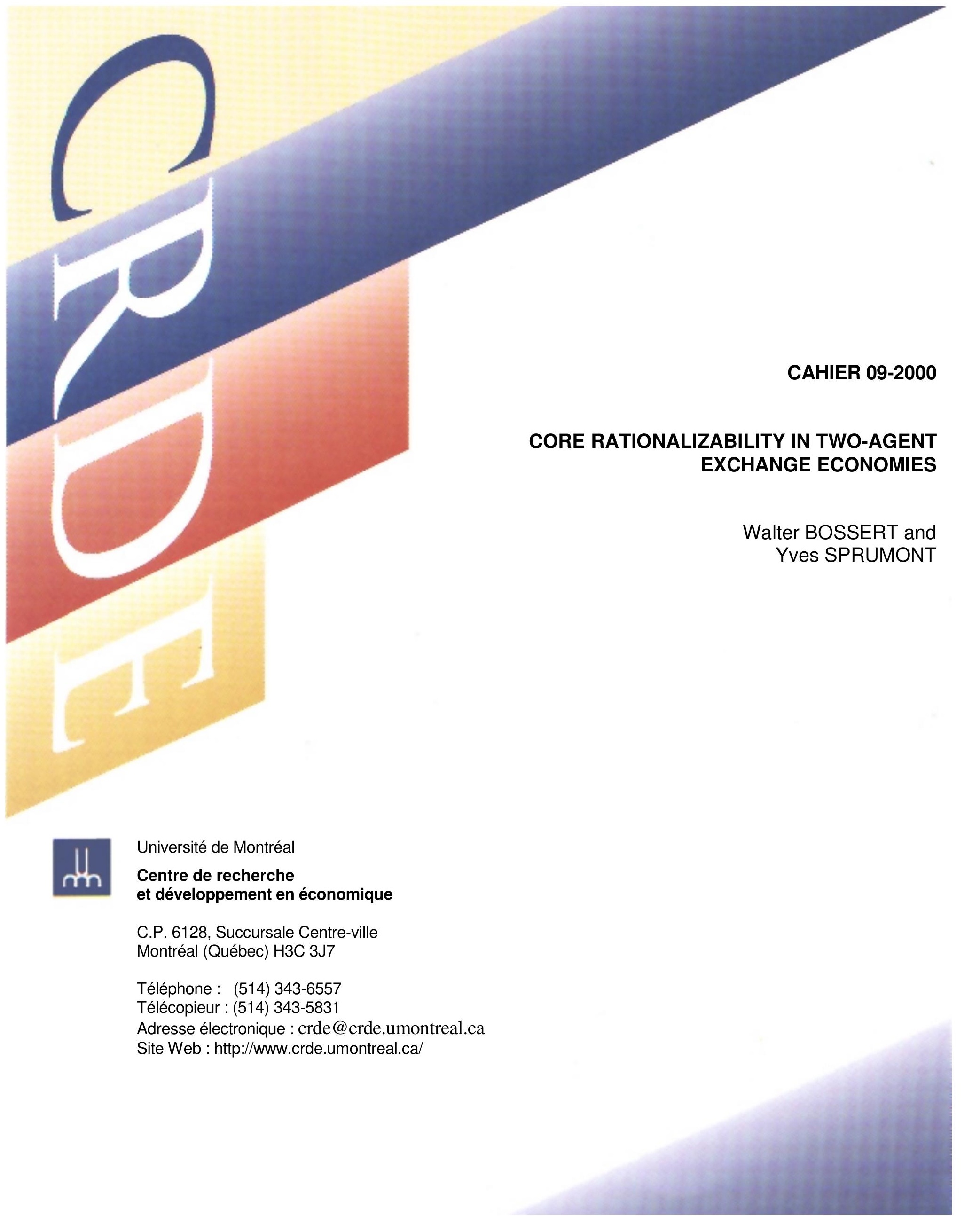


CAHIER 09-2000

\title{
CORE RATIONALIZABILITY IN TWO-AGENT EXCHANGE ECONOMIES
}

\author{
Walter BOSSERT ${ }^{1}$ and Yves SPRUMONT $^{2}$
}

1 Département de sciences économiques, Université de Montréal, and Department of Economics, Rice University

2 Centre de recherche et développement en économique (C.R.D.E.) and Département de sciences économiques, Université de Montréal

May 2000

The authors would like to thank James Redekop for valuable comments and suggestions. Financial support from the Social Sciences and Humanities Research Council (SSHRC) of Canada and the C.R.D.E. is gratefully acknowledged. 


\title{
RÉSUMÉ
}

Dans les économies d'échange à deux agents, nous caractérisons les correspondances de choix qui peuvent être rationalisées au sens du noyau.

Mots clés : rationalisation, noyaux, économies d'échange

\begin{abstract}
We provide a characterization of selection correspondences in two-person exchange economies that can be core rationalized in the sense that there exists a preference profile with some standard properties that generates the observed choices as the set of core elements of the economy for any given initial endowment vector. The approach followed in this paper deviates from the standard rational choice model in that a rationalization in terms of a profile of individual orderings rather than in terms of a single individual or social preference relation is analyzed.
\end{abstract}

Key words : collective rationality, cores, exchange economies 


\section{Introduction}

The standard approaches to rational individual choice address the question whether observed choices by economic agents can be rationalized by a single preference relation on a universal set of alternatives in the sense that these choices are given by the best elements among the feasible ones according to this rationalizing relation. Richter (1966) provides a necessary and sufficient condition for rationalizability by an ordering (a reflexive, transitive, and complete binary relation) by means of his congruence axiom. This equivalence result is obtained in a very general setting - no assumptions regarding the structure of the universal set of alternatives or the domain of the choice correspondence - the class of subsets of this set that may emerge in a choice situation - are imposed. Rationalizing relations that are not necessarily orderings are analyzed in Richter (1971), and more specific choice environments are considered, for example, in Arrow (1959), Hansson (1968), Houthakker (1950), Samuelson (1938, 1948), and Sen (1971).

Many of the results obtained regarding the rationalizability of individual choices can be applied to social choice problems as well. In that case, the issue addressed in the literature is the rationalizability of social choices by means of a single social ordering. The problem analyzed in this paper deviates from that standard framework in that we seek to investigate whether observed collective choices can be rationalized by a profile of individual preference relations rather than a single social ranking. This question is raised in Sprumont (2000) where conditions for the existence of preference profiles that generate observed choices as Nash equilibria or as the set of Pareto efficient outcomes are discussed. Brown and Matzkin (1996) derive restrictions on observed data to be generated in a general-equilibrium setting, and a revealed-preference approach to noncooperative games can be found in Zhou and Ray (1997). This paper phrases the above question in the context of an exchange economy-more specifically, we present necessary and sufficient conditions for selection correspondences to be rationalized in the sense that the observed selections are the core elements generated by individual preferences with some standard properties.

The selection correspondences analyzed here differ from the choice correspondences employed in the standard rational choice model. Consider a class of exchange economies where total endowments are fixed. A selection correspondence assigns a set of recommended allocations to each possible initial allocation of the total endowments. Thus, unlike in the case of a standard choice problem, the set of feasible options is the same for all choice situations - the selection correspondence depends on the reference point given 
by the initial endowment rather than the set of feasible allocations. As a consequence, the axioms we employ are of a different nature than those that can be found in the earlier rational choice literature.

A general analysis of this kind of collective rationalizability is a complex problem. Sprumont (1999) examines properties of the Pareto relation in the two-agent case, and the general case involving an arbitrary number of agents remains an open question. Related literature on the mathematical problem of determining the dimension of partially ordered sets confirms that general results are difficult to obtain; see, for example, Dushnik and Miller (1941), Kelly (1977, 1981), Trotter and Bogart (1976). Analogous difficulties emerge in the context of our problem and, as a consequence, our analysis in this paper is restricted to two-agent exchange economies as well. In that case, the core coincides with the set of Pareto efficient and individually rational allocations, which simplifies matters substantially. Core rationalizability (or even rationalizability of the set of Pareto efficient and individually rational allocations) in the general $n$-agent case remains an open problem. However, the number of goods need not be restricted in our results.

The remainder of the paper is organized as follows. Section 2 introduces our basic notation and definitions. In Section 3, we define the axioms that are used in our characterization of core rationalizability. Section 4 contains our main result - a theorem establishing that these axioms are necessary and sufficient for the core rationalizability of a selection correspendence in a two-agent exchange economy. The independence of the axioms is established in Section 5, and Section 6 concludes.

\section{Notation and Definitions}

Let $\mathbb{N}$ denote the set of positive integers. For $m \in \mathbb{N}$ with $m \geq 2, \mathbb{R}^{m}\left(\mathbb{R}_{+}^{m}, \mathbb{R}_{++}^{m}\right)$ is the (nonnegative, positive) $m$-orthant. The origin of $\mathbb{R}^{m}$ is denoted by $\mathbf{0}$ (because $m$ is assumed to be fixed throughout the paper, this notation is unambiguous). Our notation for vector inequalities is $\geq,>, \gg$. Weak and strict set inclusion are denoted by $\subseteq$ and $\subset$.

Suppose there are two agents in an exchange economy with $m \geq 2$ goods. The total endowments are given by $\omega \in \mathbb{R}_{++}^{m}$, and a feasible allocation is, without loss of generality, represented in terms of agent 1's consumption bundle. That is, the set of feasible allocations is given by $E=\left\{x \in \mathbb{R}_{+}^{m} \mid x \leq \omega\right\}$ where, for all $x \in E, x$ is agent 1 's consumption bundle and $\omega-x$ is the consumption of agent 2. For any $S \subseteq T \subseteq E$, $R I_{T}(S)$ is the interior of $S$ relative to $T$.

Because total endowments are fixed throughout this paper, we can, for simplicity, 
restrict attention to individual preferences defined on $E$ rather than on the entire consumption space $\mathbb{R}_{+}^{m}$. A preference ordering $R$ (with strict preference relation $P$ and indifference relation $I$ ) on $E$ is continuous if and only if the sets $\{x \in E \mid x R y\}$ and $\{x \in E \mid y R x\}$ are closed for all $y \in E$. $R$ is strictly monotonic if and only if $x>y$ implies $x P y$ for all $x, y \in E$. The relation $R$ is strictly convex if and only if, for all $x, y, z \in E$ with $x \neq y$ and all $\lambda \in(0,1), x R z$ and $y R z$ implies $(\lambda x+(1-\lambda) y) P z$. A classical exchange economy is an exchange economy where all agents' preferences are continuous, strictly monotonic, and strictly convex.

Let $R_{1}$ and $R_{2}$ be the two agents' preference orderings. For convenience, we will sometimes use agent 2's preference ordering on agent 1's consumption bundles that is induced by $R_{2}$. Specifically, this ordering $\tilde{R}_{2}$ on $E$ is defined by letting, for all $x, y \in E$, $x \tilde{R}_{2} y$ if and only if $(\omega-x) R_{2}(\omega-y)$. Clearly, each $R_{2}$ determines a unique $\tilde{R}_{2}$ and vice versa.

The set of Pareto efficient allocations for a preference profile $\left(R_{1}, R_{2}\right)$ is given by $P E\left(R_{1}, R_{2}\right)=\left\{x \in E \mid \nexists y \in E\right.$ such that $y P_{1} x$ and $\left.y \tilde{P}_{2} x\right\}$. For an initial endowment $e \in$ $E$, the set of individually rational allocations with respect to $\left(R_{1}, R_{2}\right)$ is $\operatorname{IR}\left(R_{1}, R_{2}, e\right)=$ $\left\{x \in E \mid x R_{1} e\right.$ and $\left.x \tilde{R}_{2} e\right\}$. The core of an exchange economy described by the endowment vector $e \in E$ with respect to the profile $\left(R_{1}, R_{2}\right)$ is given by $C O\left(R_{1}, R_{2}, e\right)=P E\left(R_{1}, R_{2}\right) \cap$ $\operatorname{IR}\left(R_{1}, R_{2}, e\right)$. Of course, this definition of the core as the intersection of the sets of efficient and individually rational points applies to the two-agent case only; for more than two agents, intermediate-sized coalitions have to be considered as well.

A selection correspondence is a correspondence $C: E \rightarrow E$ such that $C(e) \neq \emptyset$ for all $e \in E$. A selection correspondence selects a set of recommended allocations for each vector of initial endowments. Note that this formulation differs from the standard definition of a choice correspondence. Whereas a choice correspondence selects a (nonempty) subset of each feasible set within its domain, a selection correspondence as defined above identifies a subset of $E$ for each endowment vector, and the set of feasible allocations - the set $E$-is the same for all elements of the domain of $C$. $C$ is core rationalizable if and only if there exists a profile of continuous, strictly monotonic, and strictly convex preference orderings $\left(R_{1}, R_{2}\right)$ such that $C(e)=C O\left(R_{1}, R_{2}, e\right)$ for all $e \in E$.

Let $\emptyset \neq A \subseteq E$, and let $x, y \in A$ be such that $x \neq y$. A path from $x$ to $y$ in $A$ is a continuous function $f:[0,1] \rightarrow A$ such that $f(0)=x$ and $f(1)=y$. A path $f$ from $x$ to $y$ in $A$ is minimal if there exists no path $g$ from $x$ to $y$ in $A$ such that $g([0,1]) \subset f([0,1])$. If a path $f$ from $x$ to $y$ in $A$ is injective, it is called a simple path from $x$ to $y$ in $A$. A set $A \subseteq E$ is path connected if and only if, for all $x, y \in A$ with $x \neq y$, there exists a path 
from $x$ to $y$ in $A$. Clearly, path connectedness implies connectedness. See, for example, Brown (1968, pp. 72-76) for a discussion of different notions of connectedness.

\section{Axioms}

Because we work in a framework where a rationalization involves an entire profile of preference orderings and we require rationalizing profiles to have certain properties that are natural in the context of exchange economies, the axioms used to characterize core rationalizability are rather different from those that can be found in the standard literature on rational choice. Intuitively, the axioms used have to reflect (i) the role of the initial endowment in the economy; (ii) that the selections are consistent with the existence of a contract curve; (iii) that the selections are consistent with individual rationality; and (iv) that the preferences forming a rationalizing profile have the appropriate properties. These requirements play an essential role in the formulations of our axioms. It should be noted that, in the case of more than two agents, further properties of the core would have to be taken into account - recall that, in the two-person case, the core coincides with the set of efficient and individually rational allocations.

First, we impose a regularity condition on $C$ the interpretation of which is straightforward. It requires that the correspondence $C$ is continuous and the image of any point $e \in E$ under $C$ is a closed and connected set. Clearly, this condition is necessary for core rationalizability.

Regularity: $C$ is continuous and $C(e)$ is closed and connected for all $e \in E$.

The next axiom requires that if a point $e \in E$ is selected for some endowment vector, then it must be the unique selected point if the endowment vector is $e$ itself. Again, it is clear that a core rationalizable selection correspondence must satisfy this condition. Formally, this axiom - which we refer to as the persistence axiom - is defined as follows.

Persistence: For all $e \in C(E), C(e)=\{e\}$.

Persistence can be motivated by appealing to the interpretation of determining selected allocations in an exchange economy and the role played by the initial endowment in making that selection. A selection correspondence can be interpreted as providing a set of plausible (from a normative or descriptive viewpoint) allocations to which an economy might want to move from a given initial endowment vector. If an allocation $e$ has been revealed to have some plausibility in the sense that it is a recommended selection for at least some endowment vector, it is quite natural to postulate that, if $e$ happens to be 
the initial endowment vector, no movement away from it should be recommended. As mentioned above, the axioms used in a characterization of core rationalizability must be expected to lead towards establishing the existence of a contract curve and the requirement of individual rationality, and the persistence axiom captures aspects of these requirements.

Our next axiom is a monotonicity property. The core of a two-agent classical exchange economy is strictly monotonic with respect to the distribution of the total endowment in the following sense: from an agent's point of view, the best and worst allocations in the core get strictly better as his share of the total endowment becomes strictly larger. Because the distribution of the total endowment is the only argument of a selection correspondence, we need to translate that monotonicity property, which is necessary for core rationalizability, into one that does not rely on the knowledge of any other characteristics. To this end we introduce the following notation. For $e \in E$, let $\mathcal{S}_{0}(e)=\{S \subseteq C(E) \mid C(e) \subseteq S$ and $\mathbf{0} \in S$ and $S$ is path connected $\}$ and $\mathcal{S}_{\omega}(e)=\{S \subseteq C(E) \mid C(e) \subseteq S$ and $\omega \in S$ and $S$ is path connected $\}$. The following strict path monotonicity condition requires that the intersection of the sets in $\mathcal{S}_{0}(e)$ is strictly monotonic in $e$ with respect to set inclusion, and an analogous requirement is imposed on $\mathcal{S}_{\omega}(e)$.

Strict Path Monotonicity: For all $e, e^{\prime} \in E$ such that $e^{\prime}>e$,

$$
\bigcap_{S \in \mathcal{S}_{0}(e)} S \subset \bigcap_{S \in \mathcal{S}_{0}\left(e^{\prime}\right)} S \text { and } \bigcap_{S \in \mathcal{S}_{\omega}\left(e^{\prime}\right)} S \subset \bigcap_{S \in \mathcal{S}_{\omega}(e)} S .
$$

Finally, parallel to the role played by strict path monotonicity with respect to a monotonicity property, we define an averaging-reduction axiom which is related to the strict convexity of the rationalizing individual preferences to be established. Observe that the core of a two-agent classical exchange economy has the following property regarding the results of averaging two endowment vectors: any strict convex combination of two distinct endowment vectors leads to a core such that the worst (best) allocation in this core for each agent is strictly better (worse) than the worst (best) allocation in the union of the cores corresponding to the original two endowment vectors. Again, the corresponding condition (which is necessary for core rationalizability) has to be formulated entirely in terms of the primitives of the problem. For $e, e^{\prime} \in E$, let $\mathcal{S}\left(e, e^{\prime}\right)=\left\{S \subseteq C(E) \mid C(e) \cup C\left(e^{\prime}\right) \subseteq S\right.$ and $S$ is path connected $\}$.

Strict Averaging Reduction: For all $e, e^{\prime} \in E$ such that $e \neq e^{\prime}$, for all $\lambda \in(0,1)$,

$$
C\left(\lambda e+(1-\lambda) e^{\prime}\right) \subseteq R I_{C(E)}\left(\bigcap_{S \in \mathcal{S}\left(e, e^{\prime}\right)} S\right)
$$


The formulations of the last two axioms are somewhat involved. This is the case in order to ensure that all axioms are defined on the primitives of the problem and that each axiom can be defined on its own without reference to other properties, which is an important aspect of a proper characterization.

\section{Core-Rationalizable Selection Correspondences}

The main result of this paper is a characterization of all core-rationalizable selection correspondences by means of the axioms introduced in the previous section. Before stating and proving it, we present to lemmas that will be used in its proof. The first of those establishes a result on the relationship between minimal paths and simple paths. ${ }^{1}$

Lemma 1 Let $\emptyset \neq A \subseteq E$, and let $x, y \in A$ be such that $x \neq y$. If there exists a minimal path $f:[0,1] \rightarrow A$ from $x$ to $y$ in $A$, then there exists a simple path $g:[0,1] \rightarrow A$ from $x$ to $y$ in $A$ with $g([0,1])=f([0,1])$.

Proof. Suppose $f$ is a minimal path from $x$ to $y$ with $x, y \in A$ and $x \neq y$. The proof proceeds by constructing a simple path $g$ such that $g([0,1])=f([0,1])$.

Let $s_{0}=\max \{u \mid f(u)=x\}$ and define the function $h:[0,1] \rightarrow A$ by letting $h(u)=$ $f\left(s_{0}+u\left(1-s_{0}\right)\right)$ for all $u \in[0,1]$. Because $f$ is minimal, $h$ is a path from $x$ to $y$ in $A$ such that $h([0,1])=f([0,1])$ and $h(u) \neq x$ for all $u \in(0,1]$. Let $t_{0}=\min \{u \mid h(u)=y\}$, and define $g_{0}:[0,1] \rightarrow A$ by letting $g_{0}(u)=h\left(u t_{0}\right)$ for all $u \in[0,1]$. It follows that $g_{0}$ is a path from $x$ to $y$ in $A$ such that $g_{0}([0,1])=f([0,1]), g_{0}(u) \neq x$ for all $u \in(0,1]$, and $g_{0}(u) \neq y$ for all $u \in[0,1)$.

Next, let $s_{1}=\min \left\{u \mid g_{0}(u)=g_{0}(1 / 2)\right\}$ and $t_{1}=\max \left\{u \mid g_{0}(u)=g_{0}(1 / 2)\right\}$. By definition, $0<s_{1} \leq 1 / 2 \leq t_{1}<1$. Define $g_{1}:[0,1] \rightarrow A$ as follows.

$$
g_{1}(u)= \begin{cases}g_{0}\left(2 u s_{1}\right) & \text { if } u \in[0,1 / 2] \\ g_{0}\left(t_{1}+2(u-1 / 2)\left(1-t_{1}\right)\right) & \text { if } u \in(1 / 2,1]\end{cases}
$$

By definition, $g_{1}(u) \neq g_{1}(1 / 2)$ for all $u \in[0,1] \backslash\{1 / 2\}$. Next, we prove that $g_{1}([0,1 / 2]) \cap$ $g_{1}([1 / 2,1])=\left\{g_{1}(1 / 2)\right\}$. Clearly, $g_{1}(1 / 2)$ is in this intersection. By way of contradiction, suppose there exist $\tilde{s}_{1} \in[0,1 / 2)$ and $\tilde{t}_{1} \in(1 / 2,1]$ such that $g_{1}\left(\tilde{s}_{1}\right)=g_{1}\left(\tilde{t}_{1}\right)$. Let

$$
\tilde{g}_{1}(u)= \begin{cases}g_{1}\left(2 u \tilde{s}_{1}\right) & \text { if } u \in[0,1 / 2] \\ g_{1}\left(\tilde{t}_{1}+2(u-1 / 2)\left(1-\tilde{t}_{1}\right)\right) & \text { if } u \in(1 / 2,1]\end{cases}
$$

\footnotetext{
${ }^{1}$ The proof of this lemma was provided by James Redekop.
} 
Then $\tilde{g}_{1}$ is a path from $x$ to $y$ in $A$ such that $g_{1}(1 / 2)$ does not belong to $\tilde{g}_{1}([0,1])$. Thus, $\tilde{g}_{1}([0,1])$ is strictly included in $f([0,1])$, contradicting the minimality of $f$.

Now the above construction can be repeated for the intervals $[0,1 / 2]$ and $[1 / 2,1]$ to obtain a path $g_{2}$ from $x$ to $y$ in $A$ such that $g_{2}([0,1])=f([0,1]), g_{2}([0,1 / 2]) \cap g_{2}([1 / 2,1])=$ $\left\{g_{2}(1 / 2)\right\}, g_{2}([0,1 / 4]) \cap g_{2}([1 / 4,1 / 2])=\left\{g_{2}(1 / 4)\right\}$, and $g_{2}([1 / 2,3 / 4]) \cap g_{2}([3 / 4,1])=$ $\left\{g_{2}(3 / 4)\right\}$. This procedure can be repeated to obtain, for any $n \in \mathbb{N}$, a path $g_{n}$ from $x$ to $y$ in $A$ such that $g_{n}([0,1])=f([0,1]), g_{n}\left(\left[j / 2^{r},(j+1) / 2^{r}\right]\right) \cap g_{n}\left(\left[(j+1) / 2^{r},(j+2) / 2^{r}\right]\right)=$ $\left\{g_{n}\left((j+1) / 2^{r}\right)\right\}$ for all $r \in\{1, \ldots, n\}$ and all $j \in\left\{0, \ldots, 2^{r}-2\right\}$,

$$
g_{n}\left(\left[j / 2^{r},(j+1) / 2^{r}\right]\right) \cap g_{n}\left(\left[k / 2^{r},(k+1) / 2^{r}\right]\right)=\emptyset
$$

for all $r \in\{1, \ldots, n\}$, all $j \in\left\{0, \ldots, 2^{r}-1\right\}$ and all $k \in\left\{0, \ldots, 2^{r}-1\right\} \backslash\{j-1, j, j+1\}$, and

$$
g_{n}\left(\left[j / 2^{n-1},(j+1) / 2^{n-1}\right]\right)=g_{n-1}\left(\left[j / 2^{n-1},(j+1) / 2^{n-1}\right]\right)
$$

for all $j \in\left\{0, \ldots, 2^{n-1}-1\right\}$. Note that, for all $n \in \mathbb{N}$ and for all $u, v \in[0,1], g_{n}(u)=g_{n}(v)$ implies $|u-v|<2^{-n}$, that is, violations of injectivity are such that distinct points where $g_{n}$ assumes the same value are moving closer and closer together as $n$ increases.

For all $n \in \mathbb{N}$ and all $u \in[0,1]$, let $\bar{k}(n, u)$ be the unique integer such that $\bar{k}(n, u) / 2^{n} \leq$ $u<(\bar{k}(n, u)+1) / 2^{n}$. Define $\hat{g}(u)=\bigcap_{n \in \mathbb{N}} g_{n}\left(\left[\bar{k}(n, u) / 2^{n},(\bar{k}(n, u)+1) / 2^{n}\right]\right)$. By $(2)$ and the observation that these sets are subsets of the compact set $f([0,1]), \hat{g}(u)$ is nonempty for all $u \in[0,1]$. Next, we show that $\hat{g}(u)$ is a singleton for all $u \in[0,1]$. Consider first the case $u \in(0,1]$. Let $v<u$, and let $r, j, k$ be such that $v \in\left[j / 2^{r},(j+1) / 2^{r}\right]$ and $u \in\left[k / 2^{r},(k+1) / 2^{r}\right]$ with $k<j-1$ and $r \in\{1, \ldots, n\}$. By (1) and the definition of $\hat{g}$, it follows that $\hat{g}(v) \cap \hat{g}(u)=\emptyset$. Because $\hat{g}([0,1])=f([0,1])$ and $\hat{g}(u) \subseteq f([0,1])$, it follows that $\hat{g}(u)$ is a singleton. That $\hat{g}(0)$ is a singleton follows analogously. Therefore, we can define an injective function $g:[0,1] \rightarrow A$ by letting, for all $u \in[0,1]$ and all $a \in A$, $g(u)=a$ if and only if $\hat{g}(u)=\{a\}$. We have $g(0)=x, g(1)=y$, and $g([0,1])=f([0,1])$. That $g$ is continuous follows from the continuity of $f$ and the definition of $g$. Therefore, $g$ has all the required properties.

Our second preliminary result shows that persistence and strict path monotonicity impose some structure on the image of $E$ under $C$. In particular, those two axioms imply that $C(E)$ is the image of a simple path from $\mathbf{0}$ to $\omega$ in $C(E)$. Clearly, this property is necessary for $C(E)$ to be the contract curve for a classical exchange economy. It is interesting to note that this property does not have to be imposed explicitly in this paper - it follows as a result of other axioms. 
Lemma 2 If $C$ satisfies persistence and strict path monotonicity, then there exists a simple path $f:[0,1] \rightarrow C(E)$ from $\mathbf{0}$ to $\omega$ in $C(E)$ such that $C(E)=f([0,1])$.

Proof. First, we show that

$$
C(\mathbf{0})=\{\mathbf{0}\} \text { and } C(\omega)=\{\omega\} \text {. }
$$

Suppose, by way of contradiction, that $C(\mathbf{0}) \neq\{\mathbf{0}\}$. Because $C(\mathbf{0})$ is nonempty, there exists $x \in C(\mathbf{0})$ such that $x>\mathbf{0}$. By persistence, $C(x)=\{x\}$. Therefore, $C(x) \subseteq C(\mathbf{0})$. This implies that $\mathcal{S}_{0}(\mathbf{0}) \subseteq \mathcal{S}_{0}(x)$ and, thus,

$$
\bigcap_{S \in \mathcal{S}_{0}(x)} S \subseteq \bigcap_{S \in \mathcal{S}_{0}(\mathbf{0})} S,
$$

contradicting strict path monotonicity. That $C(\omega)=\{\omega\}$ is proven analogously.

Using (3), we next prove

$$
\mathcal{S}_{0}(\omega) \neq \emptyset .
$$

By (3), $\mathbf{0} \in C(\mathbf{0})$, which implies that $\{\mathbf{0}\} \in \mathcal{S}_{0}(\mathbf{0})$ and, thus, $\mathcal{S}_{0}(\mathbf{0})$ is nonempty. Therefore,

$$
\mathbf{0} \in \bigcap_{S \in \mathcal{S}_{0}(\mathbf{0})} S .
$$

Because $\boldsymbol{\omega}>\mathbf{0}$, strict path monotonicity implies

$$
\bigcap_{S \in \mathcal{S}_{0}(\mathbf{0})} S \subset \bigcap_{S \in \mathcal{S}_{0}(\omega)} S .
$$

By (5),

$$
\mathbf{0} \in \bigcap_{S \in \mathcal{S}_{0}(\omega)} S
$$

and, hence,

$$
\bigcap_{S \in \mathcal{S}_{0}(\omega)} S \neq \emptyset,
$$

which proves that $\mathcal{S}_{0}(\omega)$ is nonempty.

Clearly, (4) implies that there exists a path from $\mathbf{0}$ to $\omega$ in $C(E)$. This observation is now used to show that

$$
C(E)=S^{0} \text { for all } S^{0} \in \mathcal{S}_{0}(\omega) .
$$

Let $S^{0} \in \mathcal{S}_{0}(\omega)$. By definition, $S^{0} \subseteq C(E)$. To prove the converse set inclusion, let $x \in C(E)$. If $x=\mathbf{0}$ or $x=\omega, x \in S^{0}$ follows from the definition of $\mathcal{S}_{0}(\omega)$ and the fact 
that $C(\omega)=\{\omega\}$. Now suppose $\mathbf{0}<x<\omega$. By persistence, $C(x)=\{x\}$. Using strict path monotonicity, we obtain

$$
\bigcap_{S \in \mathcal{S}_{0}(\mathbf{0})} S \subset \bigcap_{S \in \mathcal{S}_{0}(x)} S \subset \bigcap_{S \in \mathcal{S}_{0}(\omega)} S \subseteq S^{0}
$$

where the last set inclusion in (7) follows because $S^{0} \in \mathcal{S}_{0}(\omega)$. This implies that

$$
\emptyset \neq \bigcap_{S \in \mathcal{S}_{0}(x)} S \subset S^{0}
$$

Because $C(x)=\{x\}$, this implies

$$
x \in \bigcap_{S \in \mathcal{S}_{0}(x)} S \subset S^{0}
$$

which completes the proof of (6). Because all elements of $\mathcal{S}_{0}(\omega)$ are equal to $C(E)$, it follows that $C(E)$ is a minimal path from 0 to $\omega$ in $C(E)$. By Lemma 1, it follows that there exists a simple path $f:[0,1] \rightarrow C(E)$ from 0 to $\omega$ in $C(E)$ such that $C(E)=f([0,1])$.

Now we can prove

Theorem $3 C$ is core rationalizable if and only if $C$ satisfies regularity, persistence, strict path monotonicity, and strict averaging reduction.

Proof. Clearly, if $C$ is core rationalizable, it satisfies the required axioms. Now suppose $C$ satisfies regularity, persistence, strict path monotonicity, and strict averaging reduction. The proof that $C$ is core rationalizable is constructive and proceeds as follows. Using Lemma 2, we define a continous and antisymmetric ordering $\succeq$ on $C(E)$. Based on this ordering $\succeq$, we define individual orderings $R_{1}$ and $R_{2}$ and show that they are continuous, strictly monotonic, and strictly convex. The proof is completed by proving that $\left(R_{1}, R_{2}\right)$ core rationalizes $C$.

By Lemma 2, there exists a simple path $f:[0,1] \rightarrow C(E)$ from 0 to $\omega$ in $C(E)$ such that $C(E)=f([0,1])$. Define the relation $\succeq$ on $C(E)$ by letting, for all $x, y \in C(E)$,

$$
x \succeq y \Leftrightarrow f^{-1}(x) \geq f^{-1}(y)
$$

Clearly, this relation is a well-defined continuous and antisymmetric ordering because the simple path $f$ is a continuous and injective function defined on a compact set.

Now we use $\succeq$ to define orderings $R_{1}$ and $\tilde{R}_{2}$ (and, thus, $R_{2}$ ). For $x \in E$, define $\min _{\succeq} C(x)$ and $\max _{\succeq} C(x)$ as follows. For all $z \in E, \min _{\succeq} C(x)=z$ if and only if 
$z \in C(x)$ and $z^{\prime} \succeq z$ for all $z^{\prime} \in C(x)$, and $\max _{\succeq} C(x)=z$ if and only if $z \in C(x)$ and $z \succeq z^{\prime}$ for all $z^{\prime} \in C(x)$. Because $C(x)$ is closed and $\succeq$ is a continuous and antisymmetric ordering, $\min _{\succeq} C(x)$ and $\max _{\succeq} C(x)$ are well-defined and unique for all $x \in E$. Now define the relations $R_{1}, \tilde{R}_{2}$, and $R_{2}$ as follows. For all $x, y \in E$,

$$
\begin{aligned}
x R_{1} y & \Leftrightarrow \min _{\succeq} C(x) \succeq \min _{\succeq} C(y) ; \\
x \tilde{R}_{2} y & \Leftrightarrow \max _{\succeq} C(y) \succeq \max _{\succeq} C(x) ; \\
x R_{2} y & \Leftrightarrow(\omega-x) \tilde{R}_{2}(\omega-y) .
\end{aligned}
$$

Clearly, these relations are orderings. We have to show that $R_{1}$ and $R_{2}$ are continuous, strictly monotonic, and strictly convex, and that $C$ is core rationalized by $\left(R_{1}, R_{2}\right)$. Because the proof for $R_{2}$ is analogous, we only establish continuity, strict monotonicity, and strict convexity for $R_{1}$.

To show that $R_{1}$ is continuous, let $x \in E$, and let $\left\langle y_{n}\right\rangle_{n \in \mathbb{N}}$ be a sequence such that $y_{n} \in E$ and $y_{n} R_{1} x$ for all $n \in \mathbb{N}$. Suppose $\left\langle y_{n}>_{n \in \mathbb{N}}\right.$ converges to $y_{0} \in E$. We have to prove that $y_{0} R_{1} x$. By definition of $R_{1}$,

$$
\min _{\succeq} C\left(y_{n}\right) \succeq \min _{\succeq} C(x)
$$

for all $n \in \mathbb{N}$. Because $C$ and $\succeq$ are continuous, the maximum theorem (see, for example, Berge, 1963, Chapter VI) implies that

$$
\min _{\succeq} C\left(y_{n}\right) \longrightarrow \min _{\succeq} C\left(y_{0}\right) .
$$

If $x P_{1} y_{0}$, it follows that $\min _{\succeq} C(x) \succ \min _{\succeq} C\left(y_{0}\right)$, which is a contradiction to (8) and (9). Therefore, because $R_{1}$ is an ordering, we must have $y_{0} R_{1} x$.

Next, we prove the strict monotonicity of $R_{1}$. For any $x \in E$, it follows from Lemma 2 that all elements of $\mathcal{S}_{\omega}(x)$ are one and the same set. Specifically, using the definition of $\succeq$,

$$
\mathcal{S}_{\omega}(x)=\left\{\left\{z \in C(E) \mid z \succeq \min _{\succeq} C(x)\right\}\right\}
$$

and, therefore,

$$
\bigcap_{S \in \mathcal{S}_{\omega}(x)} S=\left\{z \in C(E) \mid z \succeq \min _{\succeq} C(x)\right\} .
$$

Suppose $x>y$. By strict path monotonicity and (10), $\min _{\succeq} C(x) \succ \min _{\succeq} C(y)$ and, thus, $x P_{1} y$.

In order to establish strict convexity, because $R_{1}$ is continuous and strictly monotonic, it is sufficient to prove that, for all $x, y, z \in E$ with $x \neq y$ and all $\lambda \in(0,1)$, if $x I_{1} y I_{1} z$, 
then $(\lambda x+(1-\lambda) y) P_{1} z$. Analogously to the proof of strict monotonicity, it follows that

$$
\bigcap_{S \in \mathcal{S}(x, y)} S=\left\{z \in C(E) \mid \max _{\succeq}(C(x) \cup C(y)) \succeq z \succeq \min _{\succeq}(C(x) \cup C(y))\right\}
$$

for all $x, y \in E$. Let $x I_{1} y I_{1} z, x \neq y$, and $\lambda \in(0,1)$. Therefore, using (11), it follows that $\min _{\succeq} C(x)=\min _{\succeq} C(y)=\min _{\succeq} C(z) / \in R I_{C(E)}\left(\cap_{S \in \mathcal{S}(x, y)} S\right)$. By strict averaging reduction, $\min _{\succeq} C(\lambda x+(1-\lambda) y) \in R I_{C(E)}\left(\cap_{S \in \mathcal{S}(x, y)} S\right)$. Because $\succeq$ is antisymmetric, this implies $\min _{\succeq} C(\lambda x+(1-\lambda) y) \succ \min _{\succeq} C(z)$ and hence $(\lambda x+(1-\lambda) y) P_{1} z$.

Finally, to show that $\left(R_{1}, R_{2}\right)$ core rationalizes $C$, it is sufficient to prove that

$$
\begin{gathered}
C(E) \subseteq P E\left(R_{1}, R_{2}\right) \\
C(e) \subseteq I R\left(R_{1}, R_{2}, e\right) \text { for all } e \in E \\
C O\left(R_{1}, R_{2}, e\right) \subseteq C(e) \text { for all } e \in E
\end{gathered}
$$

(12) Suppose $x \in C(E)$ and $x / \in P E\left(R_{1}, R_{2}\right)$. This implies that there exists $y \in E$ such that $y P_{1} x$ and $y \tilde{P}_{2} x$. Because $x \in C(E)$, persistence implies $C(x)=\{x\}$ and, thus, $\min _{\succeq} C(x)=\max _{\succeq} C(x)=x$. Therefore, by definition of $R_{1}$ and $\tilde{R}_{2}, \min _{\succeq} C(y) \succ$ $\min _{\succeq} C(x)=\max _{\succeq} C(x) \succ \max _{\succeq} C(y)$, a contradiction.

(13) Suppose $e \in E$ and $x \in C(e)$. By definition, $\max _{\succeq} C(e) \succeq x \succeq \min _{\succeq} C(e)$. By persistence, $C(x)=\{x\}$ and, thus, $\min _{\succeq} C(x)=\max _{\succeq} C(x)=x$. Therefore, $\min _{\succeq} C(x) \succeq \min _{\succeq} C(e)$ and $\max _{\succeq} C(e) \succeq \max _{\succeq} C(x)$, which is equivalent to $x R_{1} e$ and $x \tilde{R}_{2} e$ and hence $x \in I R\left(R_{1}, R_{2}, e\right)$.

(14) Suppose $x \in P E\left(R_{1}, R_{2}\right), x R_{1} e$, and $x \tilde{R}_{2} e$. First, we show that $x \in C(E)$. By way of contradiction, suppose $x \notin \in C(E)$. Let $y, z \in C(E)$ be such that $y I_{1} x$ and $z \tilde{I}_{2} x$. $y$ and $z$ are well-defined and unique by definition of $R_{1}$ and $\tilde{R}_{2}$ and because $\succeq$ is an antisymmetric ordering. By strict convexity, there exists a hyperplane through $x$ separating the upper contour sets of $R_{1}$ and $\tilde{R}_{2}$ at $x$ (this hyperplane has a positive normal because of strict monotonicity). Thus, $y$ cannot be in the upper contour set of $\tilde{R}_{2}$ at $z$ and, hence,

$$
z \tilde{P}_{2} y \text {. }
$$

By strict monotonicity, $\mathbf{0} \tilde{R}_{2} x \tilde{I}_{2} z$. Because (i) $C(E)$ is the image of a simple path from $\mathbf{0}$ to $\omega$ in $C(E)$; (ii) $\succeq$ is an antisymmetric ordering; and (iii) $z$ is uniquely defined, it follows that $z^{\prime} \tilde{R}_{2} z$ for all $z^{\prime} \in E$ such that $z \succeq z^{\prime}$. Because $z=\max _{\succeq} C(x) \succeq \min _{\succeq} C(x)=y$, it follows that $z \succeq y$ and hence $y \tilde{R}_{2} z$, contradicting (15). Therefore, the assumption $x \notin \in C(E)$ is false and we must have $x \in C(E)$. Consequently,

$$
C(x)=\{x\}
$$


by persistence. By definition of $R_{1}$ and $\tilde{R}_{2}, \min _{\succeq} C(x) \succeq \min _{\succeq} C(e)$ and $\max _{\succeq} C(e) \succeq$ $\max _{\succeq} C(x)$. Together with (16), it follows that $\max _{\succeq} C(e) \succeq x \succeq \min _{\succeq} C(e)$. Because $C(e)$ is a connected subset of $C(E)$ and $x \in C(E)$, this implies $x \in C(e)$.

The individual preference orderings constructed in the proof of Theorem 1 are uniquethat is, if a selection correspondence is core rationalizable, there is only one profile of preference orderings that generates the selected allocations as the core elements. This means that our notion of core rationalizability provides a way of recovering individual preferences from observed collective selections without having to observe prices.

\section{Independence of the Axioms}

The axioms used in Theorem 1 are independent. For each of the following examples, the axiom that is violated is indicated and the remaining axioms of Theorem 1 are satisfied.

Regularity. (i) Continuity of $C$. Let $R_{1}$ and $R_{2}$ be such that $R_{2}$ is continuous, strictly monotonic, and convex, $R_{1}$ is strictly monotonic and strictly convex and continuous 'almost everywhere,' except at a 'pole' located off the contract curve. See, for example, Hurwicz and Richter (1971) for a discussion of poles. Let, for all $e \in E, C(e)=C O\left(R_{1}, R_{2}, e\right)$.

(ii) Closedness of $C(e)$ for all $e \in E$. Let $R_{1}$ and $R_{2}$ be identical continuous, strictly monotonic, and strictly convex preferences. Let $C(e)=R I_{C(E)}\left(C O\left(R_{1}, R_{2}, e\right)\right)$ if $e$ is not on the contract curve, and $C(e)=\{e\}$ if $e$ is on the contract curve.

(iii) Connectedness of $C(e)$ for all $e \in E$. Let $R_{1}$ and $R_{2}$ be identical continuous, strictly monotonic, and strictly convex preferences generating a linear contract curve. If $e$ is not on the contract curve, divide $C O\left(R_{1}, R_{2}, e\right)$ into three equal-length segments and let $C(e)$ be the union of the two closed segments containing the extreme points of the core. If $e$ is on the contract curve, let $C(e)=\{e\}$.

Persistence. Let $R_{1}$ and $R_{2}$ be identical continuous, strictly monotonic, and strictly convex preferences generating a linear contract curve. For all $e \in E$, let $C(e)$ be the closed line segment on the contract curve connecting the half-way point between the core and $\mathbf{0}$ and the half-way point between the core and $\omega$.

Strict path monotonicity. Let $R_{1}$ and $R_{2}$ be identical continuous, strictly convex, but not monotonic preferences, and let $C(e)=C O\left(R_{1}, R_{2}, e\right)$ for all $e \in E$.

Strict averaging reduction. For all $e \in E$, let $C(e)$ be the set consisting of the unique point of intersection of the line passing through $\mathbf{0}$ and $\omega$ and the hyperplane $\left\{e^{\prime} \in E \mid \sum_{i=1}^{m} e_{i}^{\prime}=\sum_{i=1}^{m} e_{i}\right\}$. 


\section{Concluding Remarks}

This paper provides an analysis of collective rational choice in a two-person exchange economy. In particular, we examine the rationalizability of selection correspondences in terms of the core. As is the case for many related questions (see, for example, Dushnik and Miller, 1941, and Sprumont, 1999), our results are restricted to the two-agent case. We realize that this restriction imposes serious limitations on the applicability of our results but given the difficulties involved in obtaining characterization results in higher dimensions for that type of problem, we hope that this paper will be of use as a starting point for further investigations into this and related topics.

\section{References}

Arrow, K.J. (1959), "Rational choice functions and orderings," Economica 26, 121-127. Berge, C. (1963), Topological Spaces, Oliver \& Boyd, Edinburgh and London.

Brown, D. And Matzkin, R. (1996), "Testable restrictions on the equilibrium manifold," Econometrica 64, 1249-1262.

Brown, R. (1968), Elements of Modern Topology, McGraw-Hill, London.

Dushnik, B. AND Miller, E.W. (1941), "Partially ordered sets," American Journal of Mathematics 63, 600-610.

Hansson, B. (1968), "Choice structures and preference relations," Synthese 18, 443-458.

Houthakker, H.S. (1950), "Revealed preference and the utility function," Economica 17, 159-174.

Hurwicz, L. And Richter, M.K. (1971), "Revealed preference without demand continuity assumptions," in: Chipman, J.S., Hurwicz, L., Richter, M.K., and SonNenschein, H.F. (eds.), Preferences, Utility, and Demand, Harcourt Brace Jovanovich, New York, pp. 59-76.

Kelly, D. (1981), "On the dimension of partially ordered sets," Discrete Mathematics 35, 135-156.

Richter, M.K. (1966), "Revealed preference theory," Econometrica 34, 635-645.

Richter, M.K. (1971), "Rational choice," in: Chipman, J.S., Hurwicz, L., Richter, M.K., And Sonnenschein, H.F. (eds.), Preferences, Utility, and Demand, Harcourt Brace Jovanovich, New York, 29-58. 
SAmuelson, P.A. (1938), "A note on the pure theory of consumer's behaviour," Economica $5,61-71$.

SAmuelson, P.A. (1948), "Consumption theory in terms of revealed preference," Economica 15, 243-253.

SEn, A. (1971), "Choice functions and revealed preference," Review of Economic Studies 38, 307-317.

Sprumont, Y. (1999), "Paretian quasi-orders: two agents," mimeo, C.R.D.E., Université de Montréal.

Sprumont, Y. (2000), "On the testable implications of collective choice theories," Journal of Economic Theory, forthcoming.

Trotter, W.T. and Bogart, K.P. (1976), "On the complexity of posets," Discrete Mathematics 16, 71-82.

Zhou, L. And RAy, I. (1997), "Game theory via revealed preference," mimeo, Department of Economics, Duke University. 